\title{
Fractional Calculus for Certain Integral Operator Involving Logarithmic Coefficients
}

\author{
${ }^{1}$ Aabed Mohammed, ${ }^{1}$ Maslina Darus and ${ }^{2}$ Daniel Breaz \\ ${ }^{1}$ Faculty of Science and Technology, School of Mathematical Sciences, \\ University Kebangsaan Malaysia, 43600 Bangi, Selangor D. Ehsan, Malaysia \\ ${ }^{2}$ Department of Mathematics and Informatics, Faculty of Science, \\ University of Alba Iulia, Decembrie 1918510009 Alba Iulia, Romania
}

\begin{abstract}
Problem statement: Some properties of certain integral operators on some subclasses were studied. Approach: Certain classes defined by integral operators were introduced. The well known definitions and preliminaries results were stated. Results: Having new integral operator, the characterization problems were discussed. Thus sufficient conditions were given. Conclusion: Therefore, by having new integral operators, sufficient conditions were determined. In fact, other properties from this class could be obtained.
\end{abstract}

Key words: Univalent functions, fractional calculus, logarithmic coefficients, integral operators

\section{INTRODUCTION}

The study of integral operators has been rapidly investigated by many authors in the field of univalent functions. Recently, various integral operators have been introduced for certain class of analytic univalent functions in the unit disk. In this study, we follow the similar approach by introducing a logarithmic coefficients of analytic functions in the punctured disk. We begin by giving some well-known notations and preliminary results on the class defined by integral operators and also the basic knowledge of logarithmic. Later we derive the integral operator aforementioned. Once the integral operator being derived, we shall discuss on the sufficient conditions of certain classes defined. by:

Let denote by A the class of functions f normalized

$$
\mathrm{f}(\mathrm{z})=\mathrm{z}+\sum_{\mathrm{n}=2}^{\infty} \mathrm{a}_{\mathrm{n}} \mathrm{z}^{\mathrm{n}}
$$

Which are analytic in the open unit disk:

$$
\mathrm{U}=\{\mathrm{z}: \mathrm{z} \in \mathrm{C}|\mathrm{z}|<1\}
$$

We also denote by $\mathrm{S}$ the subclass of A consisting of functions which are also univalent in $U$. Furthermore, we denote by $\mathrm{T}$ the subclass of $\mathrm{S}$ consisting of functions whose nonzero coefficients, from the second one, are negative and normalized by:

$$
f(z)=z-\sum_{n=2}^{\infty} a_{n} z^{n}
$$

Associated with each function $\mathrm{f}$ in $\mathrm{S}$ are its logarithmic coefficients $\gamma_{n}$ defined by:

$$
\log \frac{\mathrm{f}(\mathrm{z})}{\mathrm{z}}=2 \sum_{\mathrm{n}=1}^{\infty} \gamma_{\mathrm{n}} \mathrm{z}^{\mathrm{n}}, \quad|\mathrm{z}|<1
$$

The numbers $\gamma_{n}$ are called the logarithmic coefficients of $\mathrm{f}^{[9]}$.

If $\mathrm{f}(\mathrm{z})=\mathrm{z}+\sum_{\mathrm{n}=2}^{\infty} \mathrm{a}_{\mathrm{n}} \mathrm{z}^{\mathrm{n}} \in \mathrm{S}$ then $\gamma_{1}=\frac{1}{2} \mathrm{a}_{2}$. Now, since $\left|\mathrm{a}_{2}\right| \leq 2$ and $\left|\gamma_{1}\right| \leq 1$ the inequality $\left|\gamma_{\mathrm{n}}\right| \leq \frac{1}{\mathrm{n}}$ holds for functions $f \in \mathrm{S}^{*}$, but is false for the full class $\mathrm{S}$.

In particular case, the Koebe function $\mathrm{k}(\mathrm{z})=\frac{\mathrm{z}}{(1-\mathrm{z})^{2}}=\sum_{\mathrm{n}=1}^{\infty} \mathrm{nz}$ has logarithmic coefficients:

$$
\gamma_{\mathrm{n}}=\frac{1}{\mathrm{n}},(\mathrm{n}=1,2,3, \ldots)
$$

We next define the following fractional calculus (fractional integrals and fractional derivatives) given by Owa and Srivastava ${ }^{[7,8]}$. 
Definition 1: The fractional integral of order $\lambda$ is defined for $\mathrm{f}(\mathrm{z}) \in \mathrm{A}$ by:

$$
\mathrm{D}_{z}^{-\lambda} \mathrm{f}(\mathrm{z})=\frac{1}{\Gamma(\lambda)} \int_{0}^{z} \frac{\mathrm{f}(\zeta)}{(\mathrm{z}-\zeta)^{1-\lambda}} \mathrm{d} \zeta \quad(\lambda>0)
$$

where, the multiplicity of $(\mathrm{z}-\zeta)^{\lambda-1}$ is removed by requiring $(\mathrm{z}-\zeta)$ to be real when $(\mathrm{z}-\zeta)>0$.

Definition 2: The fractional derivative of order $\lambda$ is defined for $\mathrm{f}(\mathrm{z}) \in \mathrm{A}$ by:

$$
\begin{aligned}
D_{z}{ }^{\lambda} f(z)= & \frac{d}{d z}\left(D_{z}{ }^{\lambda} f(z)\right) \\
= & \frac{1}{\Gamma(1-\lambda)} \frac{d}{d z} \int_{0}^{z} \frac{f(\zeta)}{(z-\zeta)^{\lambda}} d \zeta, \\
& (0 \leq \lambda<1)
\end{aligned}
$$

where, the multiplicity of $(z-\zeta)^{-\lambda}$ is removed by requiring $(\mathrm{z}-\zeta)$ to be real when $(\mathrm{z}-\zeta)>0$.

Definition 3: Under the hypothesis of definition 2, the fractional derivative of $m+\lambda$ is defined for a function $\mathrm{f}$ $(\mathrm{z}) \in \mathrm{A}$ by:

$$
\mathrm{D}_{\mathrm{z}}^{\mathrm{m}+\lambda} \mathrm{f}(\mathrm{z})=\frac{\mathrm{d}^{\mathrm{m}}}{\mathrm{dz}^{\mathrm{m}}}\left(\mathrm{D}_{\mathrm{z}}^{\lambda} \mathrm{f}(\mathrm{z})(0 \leq \lambda>1 ; \mathrm{m}=0,1,2, \ldots)\right.
$$

Remark 1: From definitions 1, 2 and 3, we see that:

$$
\mathrm{D}_{\mathrm{z}}^{-\lambda} \mathrm{z}^{\mathrm{n}}=\frac{\Gamma(\mathrm{n}+1)}{\Gamma(\mathrm{n}+\lambda+1)} \mathrm{z}^{\mathrm{n}+\lambda} \quad(\lambda>0)
$$

and

$$
\mathrm{D}_{z}^{\mathrm{m}+\lambda} \mathrm{Z}^{\mathrm{n}}=\frac{\Gamma(\mathrm{n}+1)}{\Gamma(\mathrm{n}-\mathrm{m}-\lambda+1)} \mathrm{z}^{\mathrm{n}-\mathrm{m} \lambda \lambda} \quad(0 \leq \lambda<1, \mathrm{~m}=0,1,2, \ldots)
$$

Therefore, we say that:

$$
\mathrm{D}_{\mathrm{z}}{ }^{\lambda} \mathrm{z}^{\mathrm{n}}=\frac{\Gamma(\mathrm{n}+1)}{\Gamma(\mathrm{n}-\lambda+1)} \mathrm{z}^{\mathrm{n}-\lambda} \quad(\lambda>0)
$$

for any real $\lambda$.

Consider the following integral operator:

$$
F_{m}(z)=\int_{0}^{z}\left(\frac{f_{1}(t)}{t}\right)^{\alpha_{1}} \cdots\left(\frac{f_{m}(t)}{t}\right)^{\alpha_{m}} d t
$$

where, $f_{i}(z) \in A$ and $\alpha_{i}>0$, for all $i \in\{1,2,3, \ldots \ldots m\}$.

These operators are introduced by Breaz and Breaz $^{[3]}$ and studied by Breaz ${ }^{[1,2]}$ and Breaz, Owa and Breaz $^{[4]}$.

\section{MATERIALS AND METHODS}

Next we state two known definitions that lead to our definitions:

Definition 4: A function $\mathrm{f} \in \mathrm{A}$ is said to be in the class $\mathrm{KD}(\mu \beta)$, if satisfies the following inequality ${ }^{[6]}$ :

$$
\operatorname{Re}\left(1+\frac{\mathrm{zf}^{\prime \prime}(\mathrm{z})}{\mathrm{f}^{\prime}(\mathrm{z})}\right) \geq \mu\left|\frac{\mathrm{z \textrm {f } ^ { \prime \prime } ( \mathrm { z } )}}{\mathrm{f}^{\prime}(\mathrm{z})}\right|+\beta
$$

for some $\mu \geq 0$ and $0 \leq \beta<1$.

Definition 5: A function $f \in T$ is said to be in the class $(\mathrm{K}, \mathrm{A}, \mathrm{B}, \mu)-\mathrm{UCV}$ iff it satisfies the condition ${ }^{[5]}$ :

$$
\left|\frac{(z-\zeta) f^{\prime \prime}(z)}{(A-B)(1-\mu) f^{\prime}(z)+B(z-\zeta) f^{\prime \prime}(z)}\right|<1
$$

where, $-1 \leq \mathrm{B}<\mathrm{A} \leq 1,-1 \leq \mathrm{B}<0,0 \leq \mu<1,|\zeta| \leq \mathrm{k}$ and all $\mathrm{z} \in \mathrm{U}$.

Now we introduce the following subclasses of $\mathrm{KD}(\mu \beta)$ and $(\mathrm{K}, \mathrm{A}, \mathrm{B}, \mu)-\mathrm{UCV}$.

Definition 6: A family of functions $\mathrm{f}_{\mathrm{i}} \in \mathrm{A}, \mathrm{i} \in\{1, \ldots, \mathrm{m}\}$ is said to be in the class $\operatorname{KDF}_{\mathrm{m}}\left(\mu, \beta, \alpha_{1}, \ldots, \alpha_{\mathrm{m}}\right)$, if satisfies the inequality:

$\operatorname{Re}\left(1+\frac{\mathrm{zF}_{\mathrm{m}}{ }^{\prime}(\mathrm{z})}{\mathrm{F}_{\mathrm{m}}{ }^{\prime}(\mathrm{z})}\right) \geq \mu\left|\frac{\mathrm{zF}_{\mathrm{m}}{ }^{\prime \prime}(\mathrm{z})}{\mathrm{F}_{\mathrm{m}}{ }^{\prime}(\mathrm{z})}\right|+\beta$

For some $\mu \geq 0$ and $0 \leq \beta<1$, where $F_{m}(z)$ is defined as in (1).

Definition 7: A family of functions $f_{i}, i \in\{1, \ldots, m\}$ is said to be in the class $\left(\mathrm{K}, \mathrm{A}, \mathrm{B}, \mu, \alpha_{1}, \ldots, \alpha_{\mathrm{m}}\right)-\mathrm{UCVF}_{\mathrm{m}}$ iff it satisfies the following condition:

$\left|\frac{(\mathrm{z}-\zeta) \mathrm{F}_{\mathrm{m}}{ }^{\prime \prime}(\mathrm{z})}{(\mathrm{A}-\mathrm{B})(1-\mu) \mathrm{F}_{\mathrm{m}}{ }^{\prime}(\mathrm{z})+\mathrm{B}(\mathrm{z}-\zeta) \mathrm{F}_{\mathrm{m}}{ }^{\prime \prime}(\mathrm{z})}\right|<1$

where, $-1 \leq \mathrm{B}<\mathrm{A} \leq 1,-1 \leq \mathrm{B}<0,0 \leq \mu<1,|\zeta| \leq \mathrm{k}$, where $\mathrm{F}_{\mathrm{m}}(\mathrm{z})$ is defined as in (1). 


\section{RESULTS}

Theorem 1: Let $\mathrm{F}_{\mathrm{m}}(\mathrm{z})$ be the integral operator defined by (1) then:

$\mathrm{D}_{\mathrm{z}}{ }^{\lambda} \mathrm{F}_{\mathrm{m}}(\mathrm{z})=\left[2 \sum_{\mathrm{i}=1}^{\mathrm{m}} \sum_{\mathrm{n}=1}^{\infty} \frac{\alpha_{\mathrm{i}} \Gamma(\mathrm{n}+1)}{\Gamma(\mathrm{n}-\lambda+2)} \gamma_{\mathrm{n}, \mathrm{i}} \mathrm{z}^{\mathrm{n}-\lambda+1}\right] \mathrm{e}^{2 \sum_{\mathrm{i}=1}^{\mathrm{m}} \sum_{\mathrm{n}=1}^{\infty} \alpha_{i} \gamma_{\mathrm{n}, \mathrm{z}^{\mathrm{n}}}}$

and

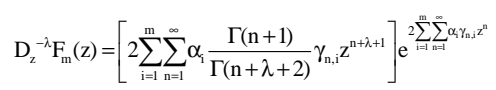

where, $D_{z}^{\lambda}$ and $D_{z}^{-\lambda}$ are the fractional derivative and fractional integral respectively of $f \in S$ and $\gamma_{n}(n=1$, $2, \ldots$ ) denote the logarithmic coefficients of $f$.

Proof: From (1) we have:

$$
F_{m}(z)=\int_{0}^{z}\left(\frac{f_{1}(t)}{t}\right)^{\alpha_{1}} \ldots\left(\frac{f_{m}(t)}{t}\right)^{\alpha_{m}} d t
$$

then

$$
\begin{aligned}
\mathrm{D}_{z}^{\lambda} \mathrm{F}_{\mathrm{m}}(\mathrm{z}) & =\mathrm{D}_{\mathrm{z}}^{\lambda-1} \mathrm{D}_{\mathrm{z}} \int_{0}^{z}\left(\frac{\mathrm{f}_{1}(\mathrm{t})}{\mathrm{t}}\right)^{\alpha_{1}} \ldots\left(\frac{\mathrm{f}_{\mathrm{m}}(\mathrm{t})}{\mathrm{t}}\right)^{\alpha_{\mathrm{m}}} \mathrm{dt} \\
& =\mathrm{D}_{\mathrm{z}}^{\lambda-1}\left[\left(\frac{\mathrm{f}_{1}(\mathrm{z})}{\mathrm{z}}\right)^{\alpha_{1}} \ldots\left(\frac{\mathrm{f}_{\mathrm{m}}(\mathrm{z})}{\mathrm{z}}\right)^{\alpha_{\mathrm{m}}}\right] \\
& =\mathrm{D}_{\mathrm{z}}^{\lambda-1} \mathrm{e}^{\log \left[\left(\frac{\mathrm{f}_{\mathrm{1}}(\mathrm{z})}{\mathrm{z}}\right)^{\alpha_{1}} \ldots\left(\frac{\mathrm{f}_{\mathrm{m}}(\mathrm{z})}{\mathrm{z}}\right)^{\alpha_{\mathrm{m}}}\right]} \\
& =\mathrm{D}_{\mathrm{z}}^{\lambda-1} \mathrm{e}^{\left[\alpha_{1} \log \frac{\mathrm{f}_{1}(\mathrm{z})}{\mathrm{z}}+\ldots+\alpha_{\mathrm{m}} \log \frac{\mathrm{f}_{\mathrm{m}}(\mathrm{z})}{\mathrm{z}}\right]}
\end{aligned}
$$

Since $\mathrm{f}_{\mathrm{i}}(\mathrm{z}) \in \mathrm{S}$, then by using logarithmic coefficients $\gamma_{n}$, we get:

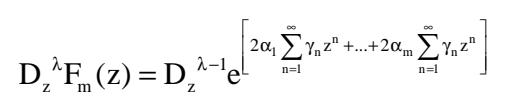

Then:

$$
\begin{aligned}
& \left.\mathrm{D}_{\mathrm{z}}^{\lambda} \mathrm{F}_{\mathrm{m}}(\mathrm{z})=\mathrm{D}_{\mathrm{z}}^{\lambda-1} \mathrm{e}^{\left[2 \sum_{\mathrm{i}=1}^{\mathrm{m}} \sum_{\mathrm{n}=1}^{\infty} \alpha_{i} \gamma_{\mathrm{r} ;} \mathrm{z}^{\mathrm{n}}\right.}\right] \\
& =\mathrm{e}^{\left[2 \sum_{\mathrm{i}=1}^{\mathrm{m}} \sum_{\mathrm{n}=1}^{\infty} \alpha_{\mathrm{i}} \gamma_{\mathrm{i}, \mathrm{z}^{\mathrm{n}}}\right]} \mathrm{D}_{\mathrm{z}}^{\lambda-1} 2 \sum_{\mathrm{i}=1}^{\mathrm{m}} \sum_{\mathrm{n}=1}^{\infty} \alpha_{\mathrm{i}} \gamma_{\mathrm{n}, \mathrm{i}} \mathrm{z}^{\mathrm{n}} \\
& \left.=\left[2 \sum_{\mathrm{i}=1}^{\mathrm{m}} \sum_{\mathrm{n}=1}^{\infty} \alpha_{\mathrm{i}} \frac{\Gamma(\mathrm{n}+1)}{\Gamma(\mathrm{n}-\lambda+2)} \gamma_{\mathrm{n}, \mathrm{i}} \mathrm{z}^{\mathrm{n}-\lambda+1}\right] \mathrm{e}^{\left[2 \sum_{\mathrm{i}=1}^{\mathrm{m}} \sum_{\mathrm{n}=1}^{\infty} \alpha_{i} \gamma_{\mathrm{n}, \mathrm{z}} \mathrm{z}^{\mathrm{n}}\right.}\right]
\end{aligned}
$$

Hence:

$$
\mathrm{D}_{\mathrm{z}}{ }^{\lambda} \mathrm{F}_{\mathrm{m}}(\mathrm{z})=\left[2 \sum_{\mathrm{i}=1}^{\mathrm{m}} \sum_{\mathrm{n}=1}^{\infty} \alpha_{\mathrm{i}} \frac{\Gamma(\mathrm{n}+1)}{\Gamma(\mathrm{n}-\lambda+2)} \gamma_{\mathrm{n}, \mathrm{i}} \mathrm{z}^{\mathrm{n}-\lambda+1}\right] \mathrm{e}^{\left[2 \sum_{\mathrm{i}=\mathrm{n}=1}^{\mathrm{m}} \sum_{\mathrm{n}=1}^{\infty} \alpha_{\mathrm{i}, \gamma_{\mathrm{i}} \mathrm{z}^{\mathrm{n}}}\right]}
$$

By using similar method we get the result (5).

\section{DISCUSSION}

Having classes defined previously, we first give a sufficient condition for a family of functions $\mathrm{f}_{\mathrm{i}} \in \mathrm{KDF}_{\mathrm{m}}$ $\left(\mu, \beta, \alpha_{1}, \ldots, \alpha_{m}\right)$. Before embarking on the proof of our result, let us calculate the expression $\frac{\mathrm{zF}_{\mathrm{m}}{ }^{\prime \prime}(\mathrm{z})}{\mathrm{F}_{\mathrm{m}}{ }^{\prime}(\mathrm{z})}$, required for proving our result.

Recall that, from (1), we have:

$$
F_{m}(z)=\int_{0}^{z}\left(\frac{f_{1}(t)}{t}\right)^{\alpha_{1}} \ldots\left(\frac{f_{m}(t)}{t}\right)^{\alpha_{m}} d t
$$

Then:

$$
\begin{aligned}
F_{m}^{\prime}(z) & =\left(\frac{f_{1}(z)}{z}\right)^{\alpha_{1}} \ldots\left(\frac{f_{m}(z)}{z}\right)^{\alpha_{m}} \\
& =e^{\alpha_{1} \log \frac{f_{1}(z)}{z}+\ldots+\alpha_{m} \log \frac{f_{m}(z)}{z}}
\end{aligned}
$$

Since $f_{i} \quad(z) \in S$, then by using logarithmic coefficients $\gamma_{n}$, we get:

$$
F_{m}^{\prime}(z)=e^{2 \alpha_{1} \sum_{n=1}^{\infty} \gamma_{n, z^{n}}+\ldots+2 \alpha_{m} \sum_{n=1}^{\infty} \gamma_{n, m^{n}}}=e^{2 \sum_{i=1}^{m} \sum_{n=1}^{\infty} \alpha_{i} \gamma_{n, z^{2}}}
$$

Here:

$$
F^{\prime \prime}(z)=2 \sum_{i=1}^{m} \sum_{n=1}^{\infty} n \alpha_{i} \gamma_{n, i} z^{n-1} e^{2 \sum_{i=1}^{m} \sum_{n=1}^{\infty} \alpha_{\gamma_{n, ~}} z^{n}}
$$

Here:

$$
\begin{aligned}
\frac{\mathrm{zF}_{\mathrm{m}}^{\prime \prime}(\mathrm{z})}{\mathrm{F}_{\mathrm{m}}^{\prime}(\mathrm{z})} & = \\
\frac{\mathrm{zF}^{\prime \prime}(\mathrm{z})}{\mathrm{F}^{\prime}(\mathrm{z})} & =\frac{2 \sum_{\mathrm{i}=1}^{\mathrm{m}} \sum_{\mathrm{n}=1}^{\infty} \mathrm{n} \alpha_{\mathrm{i}} \gamma_{\mathrm{n}, \mathrm{i}} \mathrm{z}^{\mathrm{n}} \mathrm{e}^{2 \sum_{\mathrm{i}=\mathrm{n}}^{\mathrm{m}} \sum_{\mathrm{n}=1}^{\infty} \alpha_{i} \gamma_{\mathrm{i}, \mathrm{z}^{\mathrm{n}}}}}{\sum_{\mathrm{i}=\mathrm{n}=1}^{\mathrm{m}} \sum_{\mathrm{n}=1}^{\infty} \mathrm{n} \alpha_{\mathrm{i}, \mathrm{i}} \mathrm{z}^{\mathrm{n}}} \\
& =2 \sum_{\mathrm{i}=1}^{\mathrm{m}} \sum_{\mathrm{n}=1}^{\infty} \mathrm{n} \alpha_{\mathrm{i}} \gamma_{\mathrm{n}, \mathrm{i}} \mathrm{z}^{\mathrm{n}}
\end{aligned}
$$


Theorem 2: Let the function $\mathrm{f}_{\mathrm{i}} \in \mathrm{T}$ for $\mathrm{i} \in\{1, \ldots \mathrm{m}\}$. Then the functions $\mathrm{f}_{\mathrm{i}} \in \mathrm{KDF}_{\mathrm{m}}\left(\mu, \beta, \alpha_{1}, \ldots, \alpha_{\mathrm{m}}\right)$ for $\mathrm{i} \in\{1, \ldots \mathrm{m}\}$ if and only if:

$$
\sum_{i=1}^{m} \sum_{n=1}^{\infty} 2 \alpha_{i}(\mu-1) n \gamma_{n, i} \leq 1-\beta
$$

where, $\mu \geq 0,0 \leq \beta<1$ and $\alpha_{i}>0$ for $i \in\{1, \ldots m\}$.

Proof: First, consider:

$$
\mu\left|\frac{\mathrm{zF}_{\mathrm{m}}{ }^{\prime \prime}(\mathrm{z})}{\mathrm{F}_{\mathrm{m}}{ }^{\prime}(\mathrm{z})}\right|-\operatorname{Re}\left(1+\frac{\mathrm{zF}_{\mathrm{m}}{ }^{\prime}(\mathrm{z})}{\mathrm{F}_{\mathrm{m}}{ }^{\prime}(\mathrm{z})}\right) \leq(\mu+1)\left|\frac{\mathrm{zF}_{\mathrm{m}}{ }^{\prime \prime}(\mathrm{z})}{\mathrm{F}_{\mathrm{m}}{ }^{\prime}(\mathrm{z})}\right|
$$

From (6) we obtain:

$$
\begin{aligned}
(\mu+1)\left|\frac{\mathrm{zF}_{\mathrm{m}}{ }^{\prime \prime}(\mathrm{z})}{\mathrm{F}_{\mathrm{m}}{ }^{\prime}(\mathrm{z})}\right|= & (\mu+1)\left|2 \sum_{\mathrm{i}=1}^{\mathrm{m}} \sum_{\mathrm{n}=1}^{\infty} \mathrm{n} \alpha_{\mathrm{i}} \gamma_{\mathrm{n}, \mathrm{i}} \mathrm{z}^{\mathrm{n}}\right| \\
& \leq 2(\mu+1) \sum_{\mathrm{i}=1}^{\mathrm{m}} \sum_{\mathrm{n}=1}^{\infty} \mathrm{n} \alpha_{\mathrm{i}}\left|\gamma_{\mathrm{n}, \mathrm{i}}\right|\left|\mathrm{z}^{\mathrm{n}}\right| \\
& \leq 2(\mu+1) \sum_{\mathrm{i}=1}^{\mathrm{m}} \sum_{\mathrm{n}=1}^{\infty} \mathrm{n} \alpha_{\mathrm{i}} \gamma_{\mathrm{n}, \mathrm{i}}
\end{aligned}
$$

If (7) holds then the above expression is bounded by $1-\beta$ and consequently:

$$
\mu\left|\frac{z F_{m}{ }^{\prime \prime}(z)}{F_{m}{ }^{\prime}(z)}\right|-\operatorname{Re}\left(1+\frac{z F_{m}^{\prime \prime}(z)}{F_{m}{ }^{\prime}(z)}\right)<-\beta
$$

Which equivalent to:

$$
\operatorname{Re}\left(1+\frac{\mathrm{zF}_{\mathrm{m}}{ }^{\prime \prime}(\mathrm{z})}{\mathrm{F}_{\mathrm{m}}{ }^{\prime}(\mathrm{z})}\right) \geq \mu\left|\frac{\mathrm{zF}_{\mathrm{m}}{ }^{\prime \prime}(\mathrm{z})}{\mathrm{F}_{\mathrm{m}}{ }^{\prime}(\mathrm{z})}\right|+\beta
$$

Hence $\mathrm{f}_{\mathrm{i}} \in \mathrm{KDF}_{\mathrm{m}}\left(\mu, \beta, \alpha_{1}, \ldots, \alpha_{\mathrm{m}}\right)$. for $\mathrm{i} \in\{1, \ldots \mathrm{m}\}$.

Conversely, let $\mathrm{f}_{\mathrm{i}} \in \mathrm{KDF}_{\mathrm{m}}\left(\mu, \beta, \alpha_{1}, \ldots, \alpha_{\mathrm{m}}\right)$. for $\mathrm{i} \in\{1, \ldots \mathrm{m}\}$ and prove that (7) holds.

If $\mathrm{f}_{\mathrm{i}} \in \mathrm{KDF}_{\mathrm{m}}\left(\mu, \beta, \alpha_{1}, \ldots, \alpha_{\mathrm{m}}\right) \mathrm{i} \in\{1, \ldots \mathrm{m}\}$ and $\mathrm{z}$ is real, we get from (2) and (6):

$$
\begin{aligned}
& 1+2 \sum_{\mathrm{i}=1}^{\mathrm{m}} \sum_{\mathrm{n}=1}^{\infty} \mathrm{n} \alpha_{\mathrm{i}} \gamma_{\mathrm{n}, \mathrm{i}} \mathrm{z}^{\mathrm{n}} \geq \mu\left|2 \sum_{\mathrm{i}=1}^{\mathrm{m}} \sum_{\mathrm{n}=1}^{\infty} \mathrm{n} \alpha_{\mathrm{i}} \gamma_{\mathrm{n}, \mathrm{i}} \mathrm{z}^{\mathrm{n}}\right|+\beta \\
& \geq 2 \mu \sum_{\mathrm{i}=1}^{\mathrm{m}} \sum_{\mathrm{n}=1}^{\infty} \mathrm{n} \alpha_{\mathrm{i}} \gamma_{\mathrm{n}, \mathrm{i}} \mathrm{z}^{\mathrm{n}}+\beta
\end{aligned}
$$

That is equivalent to:

$$
2 \mu \sum_{\mathrm{i}=1}^{\mathrm{m}} \sum_{\mathrm{n}=1}^{\infty} \alpha_{\mathrm{i}} \gamma_{\mathrm{n}, \mathrm{i}} \mathrm{n} \mathrm{z}^{\mathrm{n}}-2 \sum_{\mathrm{i}=1}^{\mathrm{m}} \sum_{\mathrm{n}=1}^{\infty} \alpha_{\mathrm{i}} \gamma_{\mathrm{n}, \mathrm{i}} \mathrm{nz} \mathrm{z}^{\mathrm{n}} \leq 1-\beta
$$

The above inequality reduces to:

$$
\sum_{i=1}^{m} \sum_{n=1}^{\infty} 2 \alpha_{i}(\mu-1) n \gamma_{n, i} z^{n} \leq 1-\beta
$$

Let $\mathrm{z} \rightarrow 1^{-}$along the real axis, then we get:

$$
\sum_{i=1}^{m} \sum_{n=1}^{\infty} 2 \alpha_{i}(\mu-1) n \gamma_{n, i} \leq 1-\beta
$$

This gives the required result. Next, we consider the class:

$$
\mathrm{f}_{\mathrm{i}} \in \mathrm{KDF}_{\mathrm{m}}\left(\mathrm{K}, \mathrm{A}, \mathrm{B}, \mu, \beta, \alpha_{1}, \ldots, \alpha_{\mathrm{m}}\right)-\mathrm{UCVF}_{\mathrm{m}}
$$

Theorem 3: Let the functions $f_{i} \in T$. for $i \in\{1, \ldots m\}$. Then the functions $\mathrm{f}_{\mathrm{i}} \in \mathrm{KDF}_{\mathrm{m}}\left(\mathrm{K}, \mathrm{B}, \mu, \beta, \alpha_{1}, \ldots, \alpha_{\mathrm{m}}\right)$ $\mathrm{UCVF}^{\mathrm{m}}$ for $\mathrm{i} \in\{1, \ldots \mathrm{m}\}$ if and only if:

$\sum_{i=1}^{m} \sum_{n=1}^{\infty} 2(1-B)(1+k) n \alpha_{i} \gamma_{n, i}<(A-B)(1-\mu)$

where, $-1 \leq \mathrm{B}<\mathrm{A} \leq 1-1 \leq \mathrm{B}<0,0 \leq \mu<1,0 \leq \mathrm{K}<\infty$ and $\alpha_{\mathrm{i}}>0$ for $\mathrm{i} \in\{1, \ldots \mathrm{m}\}$.

Proof: Suppose that $\mathrm{f}_{\mathrm{i}} \in \mathrm{KDF}_{\mathrm{m}}\left(\mathrm{K}, \mathrm{B}, \mu, \beta, \alpha_{1}, \ldots, \alpha_{\mathrm{m}}\right)$ $\mathrm{UCVF}_{\mathrm{m}}$. Then we obtain from (3) and (6):

$$
\begin{aligned}
& \left|\frac{(\mathrm{z}-\zeta) \frac{\mathrm{F}_{\mathrm{m}}{ }^{\prime \prime}(\mathrm{z})}{\mathrm{F}_{\mathrm{m}}{ }^{\prime}(\mathrm{z})}}{(\mathrm{A}-\mathrm{B})(1-\mu)+\mathrm{B}(\mathrm{z}-\zeta) \frac{\mathrm{F}_{\mathrm{m}}{ }^{\prime \prime}(\mathrm{z})}{\mathrm{F}_{\mathrm{m}}{ }^{\prime}(\mathrm{z})}}\right| \\
& =\left|\frac{2(\mathrm{z}-\zeta) \sum_{\mathrm{i}=1}^{m} \sum_{\mathrm{n}=1}^{\infty} \mathrm{n} \alpha_{\mathrm{i}} \gamma_{\mathrm{n}, \mathrm{i}} \mathrm{z}^{\mathrm{n}-1}}{(\mathrm{~A}-\mathrm{B})(1-\mu)+2 B(\mathrm{z}-\zeta) \sum_{\mathrm{i}=1}^{m} \sum_{\mathrm{n}=1}^{\infty} n \alpha_{\mathrm{i}} \gamma_{\mathrm{n}, \mathrm{i}} \mathrm{z}^{\mathrm{n}-1}}\right|<1
\end{aligned}
$$

Since $\operatorname{Re}(\mathrm{z}) \leq|\mathrm{z}|$ for all $\mathrm{z} \in \mathrm{U}$.

Then we have:

$$
\operatorname{Re}\left(\frac{2(\mathrm{z}-\zeta) \sum_{\mathrm{i}=1}^{\mathrm{m}} \sum_{\mathrm{n}=1}^{\infty} \mathrm{n} \alpha_{\mathrm{i}} \gamma_{\mathrm{n}, \mathrm{i}} \mathrm{z}^{\mathrm{n}-1}}{(\mathrm{~A}-\mathrm{B})(1-\mu)+2 \mathrm{~B}(\mathrm{z}-\zeta) \sum_{\mathrm{i}=1}^{\mathrm{m}} \sum_{\mathrm{m}=1}^{\infty} \mathrm{n} \alpha_{\mathrm{i}} \gamma_{\mathrm{n}, \mathrm{i}} \mathrm{z}^{\mathrm{n}-1}}\right)<1
$$

If we choose $\mathrm{z}$ and $\xi$ real and letting $\mathrm{z} \rightarrow 1^{-}$and $\xi \rightarrow-$ $\mathrm{k}^{+}$, we obtain: 


$$
\frac{2(1+k) \sum_{i=1}^{m} \sum_{n=1}^{\infty} n \alpha_{i} \gamma_{n, i}}{(A-B)(1-\mu)+2 B(1+k) \sum_{i=1}^{m} \sum_{n=1}^{\infty} n \alpha_{i} \gamma_{n, i}}<1
$$

The above inequality reduces to:

$2(1+k) \sum_{i=1}^{m} \sum_{n=1}^{\infty} n \alpha_{i} \gamma_{n, i}<(A-B)(1-\mu)+2(1+k) B \sum_{i=1}^{m} \sum_{n=1}^{\infty} n \alpha_{i} \gamma_{n, i}$

This is equivalent to:

$2(1+k) \sum_{i=1}^{m} \sum_{n=1}^{\infty} n \alpha_{i} \gamma_{n, i}-2(1+k) B \sum_{i=1}^{m} \sum_{n=1}^{\infty} n \alpha_{i} \gamma_{n, i}<(A-B)(1-\mu)$

Therefore:

$$
\sum_{i=1}^{m} \sum_{n=1}^{\infty} 2(1-B)(1+k) n \alpha_{i} \gamma_{n, i}<(A-B)(1-\mu)
$$

Conversely, assume that (8) is true and $|z|=1$ and $|\zeta| \leq \mathrm{k}$ then we have:

$$
\begin{aligned}
& \left|(z-\zeta) \frac{F_{m}{ }^{\prime \prime}(z)}{F_{m}{ }^{\prime}(z)}\right|-\left|(A-B)(1-\mu)+B(z-\zeta) \frac{F_{m}{ }^{\prime}(z)}{F_{m}{ }^{\prime}(z)}\right| \\
& =\left|2(z-\zeta) \sum_{i=1}^{m} \sum_{n=1}^{\infty} n \alpha_{i} \gamma_{n, i} z^{n-1}\right| \\
& -\left|(A-B)(1-\mu)+2 B(z-\zeta) \sum_{i=1}^{m} \sum_{n=1}^{\infty} n \alpha_{i} \gamma_{n, i} z^{n-1}\right| \\
& \leq 2(1+|\zeta|) \sum_{i=1}^{m} \sum_{n=1}^{\infty} n \alpha_{i} \gamma_{n, i} \\
& -2 B(1+|\zeta|) \sum_{i=1}^{m} \sum_{n=1}^{\infty} n \alpha_{i} \gamma_{n, i}-(A-B)(1-\mu)
\end{aligned}
$$

This is equivalent to:

$$
2(1-B)(1+k) \sum_{i=1}^{m} \sum_{n=1}^{\infty} n \alpha_{i} \gamma_{n, i}-(A-B)(1-\mu)<0
$$

By hypothesis, this implies that $\mathrm{f}_{\mathrm{i}} \in\left(\mathrm{K}, \mathrm{B}, \mu, \alpha_{1}, \ldots\right.$, $\left.\alpha_{\mathrm{m}}\right)-\mathrm{UCVF}^{\mathrm{m}}$.

\section{CONCLUSION}

The integral operator defined was motivated by Breaz and the group ${ }^{[1-4]}$ This operator can be generalised further and many other results such as the coefficient estimates and distortion theorem can be obtained.

\section{ACKNOWLEDGEMENT}

The study here is fully supported by EScienceFund grant: 04-01-02-SF0425, MOSTI, Malaysia.

\section{REFERENCES}

1. Breaz, D., 2008. A convexity property for an integral operator on the class $S_{p}(\beta)$. J. Inequal. Appli., 2008: 4-4. DOI: 10.1155/2008/143869

2. Breaz, D., 2008. Certain Integral Operators on the Classes $\mathrm{M}\left(\beta_{\mathrm{i}}\right)$ and $\mathrm{N}\left(\beta_{\mathrm{i}}\right)$. J. Inequal. Appli., 2008: 4-4. DOI: $10.1155 / 2008 / 719354$

3. Breaz, D. and N. Breaz, 2002. Two integral operators. Stud. Univ. Babes Bolyai, Math., 47: 13-19. http://www.cs.ubbcluj.ro/ studia-m/

4. Breaz, D., S. Owa and N. Breaz, 2008. A new integral univalent operator. Acta Univ. Apulensis, 16: 11-16.

http://atenea.matem.unam.mx/EMIS/journals/AUA/

5. Ozlem, G.H., E. Sumer and S. Owa, 2006. Fractional calculus and some properties of $\mathrm{k}$ uniform convex functions with negative coefficients. Taiwanese J. Math., 10: 1671-1683. http://www.math.nthu.edu.tw/ tjm/myweb/FrameC onAbs.htm

6. Shams, S., S.R. Kulkarni and J.M. Jahangiri, 2004. Classes of uniformly starlike and convex functions. Int. J. Math. Sci., 55: 2959-2961. DOI: $10.1155 / \mathrm{S} 0161171204402014$

7. Srivastava, H.M. and S. Owa, 1984. An application of the fractional derivative. Math. Jap., 29: 383-389. http://www.jams.or.jp/notice/mj/mj.html.

8. Srivastava, H.M. and S. Owa, 1989. Univalent functions, Fractional Calculus and Their Applications. Halsted Press, John Wiley and Sons, Chichester, New York, ISBN: 10: 0745807011, pp: 404.

9. Duren, P.L., 1983. Univalent Functions. SpringerVerlag, New York, ISBN: 0387907955. 